\title{
Computer Simulation of Image Distortion by Atmospheric Turbulence Using Time-Series Image Data with 250-Million-Pixels
}

\author{
Yinhao $\mathrm{Li}^{1 *}$, Yutaro Iwamoto ${ }^{1}$, Katsuhisa Ogawa ${ }^{2}$, Yen-Wei Chen ${ }^{1}$ \\ ${ }^{1}$ Ritsumeikan University, Shiga, Japan. \\ ${ }^{2}$ Canon Incorporation, Kanagawa, Japan. \\ * Corresponding author. Tel.: +81-080-2913-3632; email: gr0278ps@ed.ritsumei.ac.jp \\ Manuscript submitted July 25, 2017; accepted September 10, 2017. \\ doi: 10.17706/ijcee.2018.10.1.53-61
}

\begin{abstract}
In this research, we propose a computer simulation method to simulate degraded image by atmospheric turbulence according to different photographing distance. We use parameters measured from real time-series image data with 250-million-pixels. Target images for experiments are collected according to the distance of the subject, and the atmospheric fluctuation amount of each image is calculated. We confirm that the amount of atmospheric fluctuation increases with distance. From visual observation, it is confirmed that the simulation image distorted by atmospheric turbulence using our proposed method is quite similar to the real images.
\end{abstract}

Key words: Atmospheric turbulence, computer simulation, image distortion, 250 million pixel images.

\section{Introduction}

The development of camera technology enable us shoot farther goals with higher resolution. The biggest image in Fig. 1 is taken by Canon's 250 million pixel CMOS image sensor (Fig. 2) [2], [15], and two tiny parts (approximately one ten thousandth of the whole image) are heavily distorted by atmospheric turbulence in

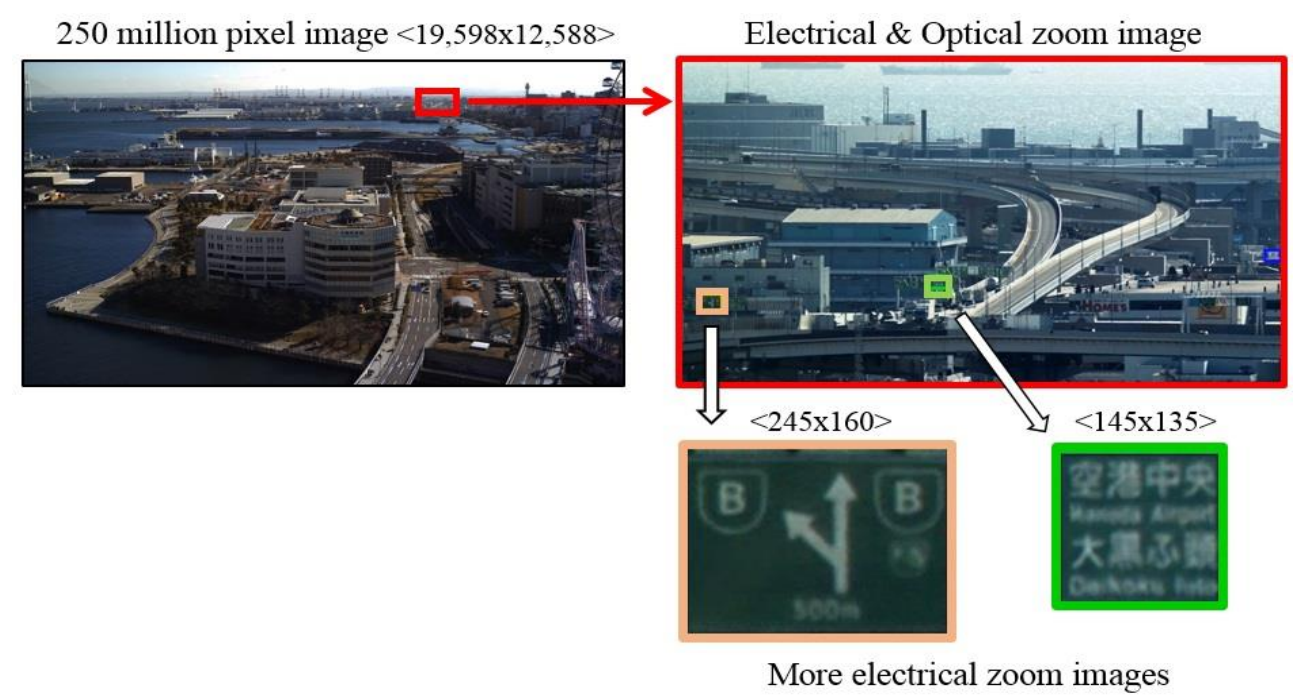

Fig. 1. Example of 250 million pixel images and expanded tiny part. Numbers near images are their size. 


\begin{tabular}{|c|c|}
\hline Number of Pixel & $19,598(\mathrm{H}) \times 12,588(\mathrm{~V})$ \\
\hline Pixel Size & $1.5 \mu \mathrm{m} \times 1.5 \mu \mathrm{m}$ \\
\hline Image Size & $\begin{array}{l}\text { APS-H-format } \\
=32.84 \mathrm{~mm} \times 25.84 \mathrm{~mm}\end{array}$ \\
\hline Frame Rate1 & $5 \mathrm{fps}=$ Full Pixel $19 \mathrm{~K} 13 \mathrm{~K}$ \\
\hline Frame Rate2 & $24 \mathrm{fps}=$ Select Pixel 8K4K \\
\hline Frame Rate 3 & $48 \mathrm{fps}=$ Select Pixel $4 \mathrm{~K} 2 \mathrm{~K}$ \\
\hline
\end{tabular}

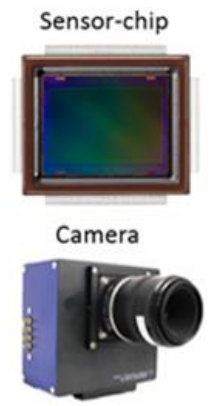

Fig. 2. 250 million pixel CMOS image sensor produced by canon.

a long distance(more than $2.5 \mathrm{~km}$ ).

In several decades, large quantities of methods are proposed to deal with the problem that restoring real images or natural images from degradation of changing and complicated atmospheric turbulence [5], [6], [9], [13], [16], [17]. Since experimental images distorted by atmospheric turbulence often include targets in a long distance, preparing a ground truth for comparing is almost impracticable. Although several image simulation models with respect to atmospheric turbulence are proposed, such as [3] and [11], the common problem of them is extremely difficult for implementation because of their complex models.

In this paper, we quantitatively analyze the degradation degree of the image in order to find the relationship between the photographing distance and the influence of atmospheric turbulence, and propose a simulation model with parameters obtained from real images to simulate the degraded image of each photographing distance. Compared with the conventional methods, our proposed method is easier to implement and suitable for quantitative evaluations of image quality improvement method. In addition, it is also a good method for data augmentation of training data in deep neural network which is currently in trend.

\section{Estimation of Atmospheric Fluctuation Amount}

We use real images (temporal sequences) to estimate the influence of atmospheric turbulence on the image. Each sequence has 300 frames and was taken by the Canon's 250 million pixel CMOS image sensor from various fixed long distances $(3.2 \mathrm{~km}, 5 \mathrm{~km}, 7 \mathrm{~km}$ and $23 \mathrm{~km})$. We choose the similar way as [13] to find out some rules of image deformation caused by atmospheric turbulence by the following procedure. (1) Extract ROI (region of interesting) from each frame. (2) Detect the SURF feature points for each frame and then find corresponding points between the first frame (reference frame) and other frames. (3) Select one feature point who has the most corresponding points in other frames. (4) Calculate the mean position of the feature point

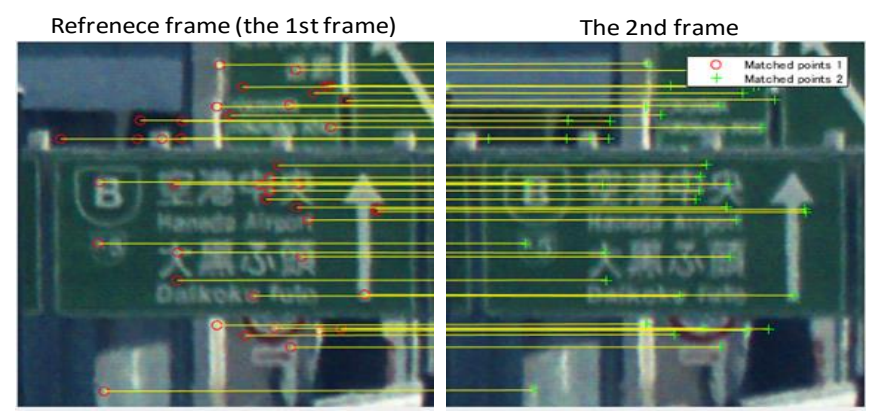

Fig. 3. Example of candidate feature point matches between the first image (reference frame) and the second image. 


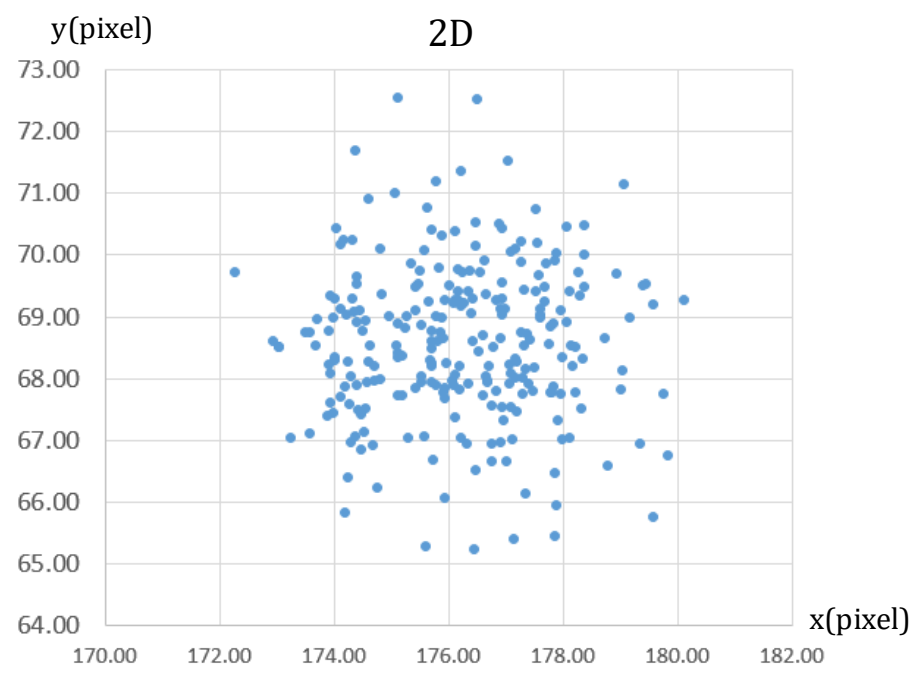

(a)
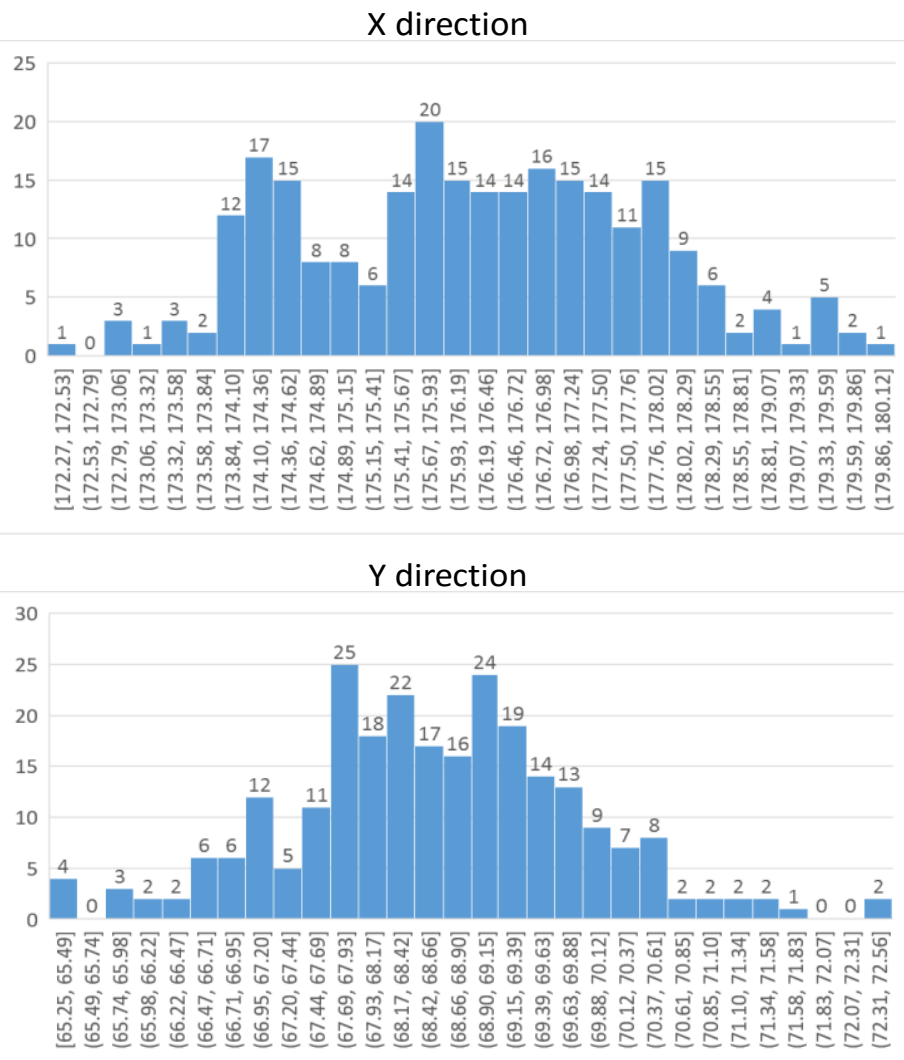

(b)

Fig. 4. (a) Spatial distribution of the selected feature point set, (b) X coordinate histogram (up) and Y coordinate histogram (down) of the selected feature point set.

over all frames. (5) Calculate the vertical and horizontal standard deviation of the position, which are used as measures of the influence of atmospheric fluctuation or simulation model parameters.

\subsection{Feature Point Detection}

We first extract ROI (region of interesting) from each frame. We detect the SURF feature points [1] for ROI of each frame because of its ease to implement and high accuracy compared to conventional SIFT [10]. Then 
we find corresponding points between the first frame (reference frame) and other 299 frames by using M-estimator SAmple Consensus (MSAC) [4], [14] and pair distance. One example of corresponding points are shown as shown in Fig. 3.

Table 1. Feature Point Distribution Test Table of Each Sample by Kolmogorov-Smirnov Test Function

\begin{tabular}{|c|c|c|}
\hline Image & $X$ direction (\%) & Y direction (\%) \\
\hline A & 100 & 100 \\
\hline B & 100 & 100 \\
\hline C & 100 & 100 \\
\hline D & 93.75 & 100 \\
\hline E & 97.96 & 100 \\
\hline F & 100 & 100 \\
\hline
\end{tabular}

\subsection{Inference of Atmospheric Turbulence}

We select one corresponding feature point set, which has the most corresponding points in other frames for analysis. The spatial distribution of the selected feature point set (which include about 250 detected feature points in original 300 frames) is shown in Fig. 4(a). Each point in Fig. 4(a) corresponds to one frame's feature point. The X coordinate histogram (up) and Y coordinate histogram (down) of the selected feature point set are shown in Fig. 4(b). By using One-sample Kolmogorov-Smirnov test [8], we confirmed both histogram can be considered as the normal distribution. We also checked all corresponding feature point sets in 6 different images. The test results (the percentage of being normal distribution) are summarized in Table 1. It can be seen that the displacements (distortions) due to the atmospheric turbulence follow the normal distribution (Gaussian distribution). So we use the statistics of the distribution to measure the inference of the atmospheric turbulence. We calculate the mean position (absolute coordinate) of the feature point over all frames. Then we calculate the mean $\left(\sigma_{\mathrm{r}}\right)$ of horizontal (X-direction) and vertical (Y-direction) standard deviations $\left(\sigma_{\mathrm{x}}\right.$ and $\sigma_{\mathrm{y}}$ ) of the position, which are used as measures of the influence of atmospheric fluctuation or simulation model parameters.

\subsection{Relationship between the Degree of Deformation and Photography Distance}

The original images with enormous size are hard to achieve and save. In this paper, we compute 4 kinds of images with different photography distances $(2.5 \mathrm{~km}, 3.2 \mathrm{~km}, 5 \mathrm{~km}, 7 \mathrm{~km}$, and $23 \mathrm{~km})$ to confirm that the degree of partial deformation aggravate as the photography distance changes. As shown in Fig. 5(a), we demonstrate partial deformation of image about the target in $23 \mathrm{~km}$ is severe compared with the target in shorter distance $(2.5 \mathrm{~km}, 3.2 \mathrm{~km}, 5 \mathrm{~km}, 7 \mathrm{~km})$.

As shown in Fig. 4 (a), the shake distribution is isotropic. Also, since there is no correlation when separating the atmospheric fluctuation component in the $\mathrm{X}$ direction and the $\mathrm{Y}$ direction, the difference between the $\mathrm{X}$ direction and $\mathrm{Y}$ direction in Fig. 5 (a) is due to measurement error. Therefore, the atmospheric fluctuation component can be expressed by only the spread (standard deviation) in the radial direction (one dimension). The relationship between the atmospheric fluctuation (the standard deviation) and the photographing distance is shown in Fig. 5(b). The point is the average of $\sigma_{\mathrm{x}}$ and $\sigma_{\mathrm{y}}$. As shown in Fig. 5 (b) the atmospheric fluctuation (the standard deviation) and the photographing distance are in a linear relation.

By the way, in the next research, we are going to set up a model, which can be used to simulate partial deformation with target in different photography distance according to the linear relationship between 


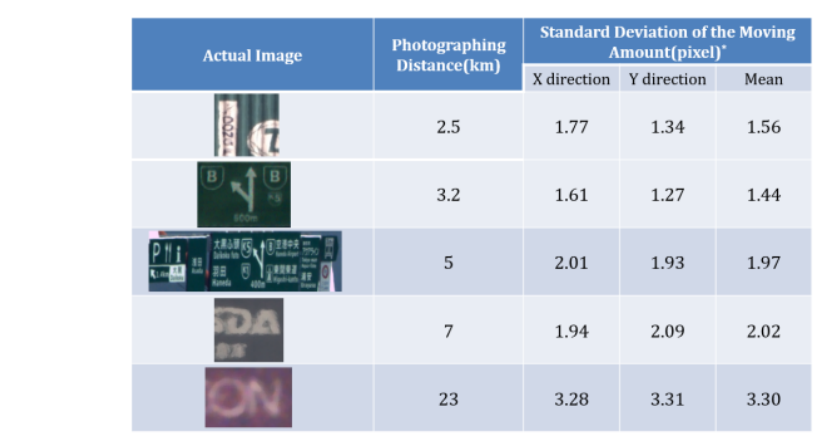

*Compute from the selected feature point in real images

(a)

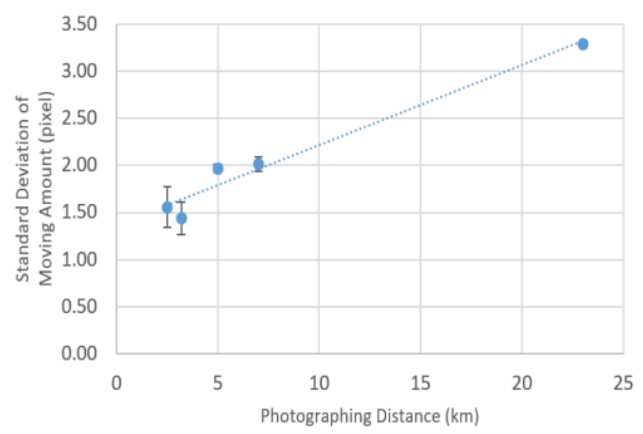

(b)

Fig. 5. (a) Standard deviation of movement about data in different photography distance, (b) Graph about linear relationship between standard deviation of moving amount (mean of X and Y direction) and photographing distance in (a). Error bars show the error between mean and standard deviation of $X$ or Y direction.

standard deviation of moving amount and photographing distance shown as Fig. 5(b). By just using the frames taken from $2.5 \mathrm{~km}, 3.2 \mathrm{~km}, 5 \mathrm{~km}, 7 \mathrm{~km}$, and $23 \mathrm{~km}$, we draw a preliminary conclusion that standard deviation of moving amount $s$ can be represented as

$$
s=0.0853 p+1.3603
$$

where $p$ is photography distance. The unit of $p$ and $s$ are pixel and kilometer respectively. The estimated line is also shown in Fig.5(b) as a dashed line.

Because of the lack of images with photographing distance arranging from $10 \mathrm{~km}$ to $20 \mathrm{~km}$, we are going to collect more images in different photographing distances for estimating a higher accurate model.

\section{Simulation Model}

Equation (2) is used to simulate $\boldsymbol{y}_{k}$, which is the $k$-th simulation frame, from a standard high-resolution image $\boldsymbol{x}$.

$$
\mathbf{y}_{k}=\boldsymbol{D} \boldsymbol{B}_{k} \boldsymbol{W}_{k} \mathbf{x}+\boldsymbol{N}_{k}
$$

where $\boldsymbol{W}_{k}$ is a warping operator to simulate the deformation by atmospheric turbulence. $\boldsymbol{B}_{k}$ is a Gaussian blur kernel. $\boldsymbol{D}$ is a decimation (down-sampling) operator with a scale of $\times 0.5$. $\boldsymbol{N}_{k}$ is a Gaussian noise. In this paper, we set the standard deviation of Gaussian noise for pixel values to be 1.275.
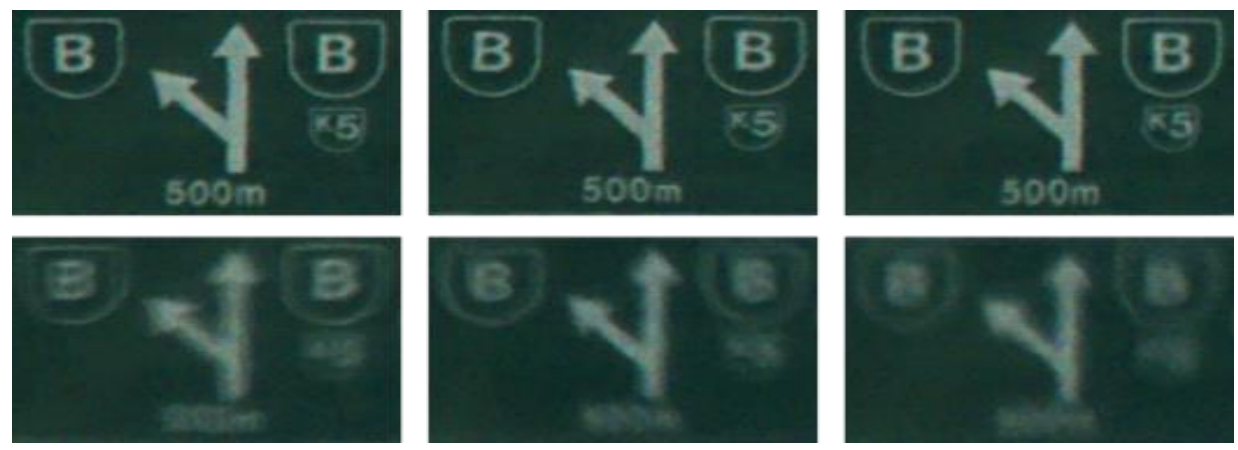

Fig. 6. Samples of initial real frames (up: three frames are relatively sharp; bottom: three frames are not clear at all). 

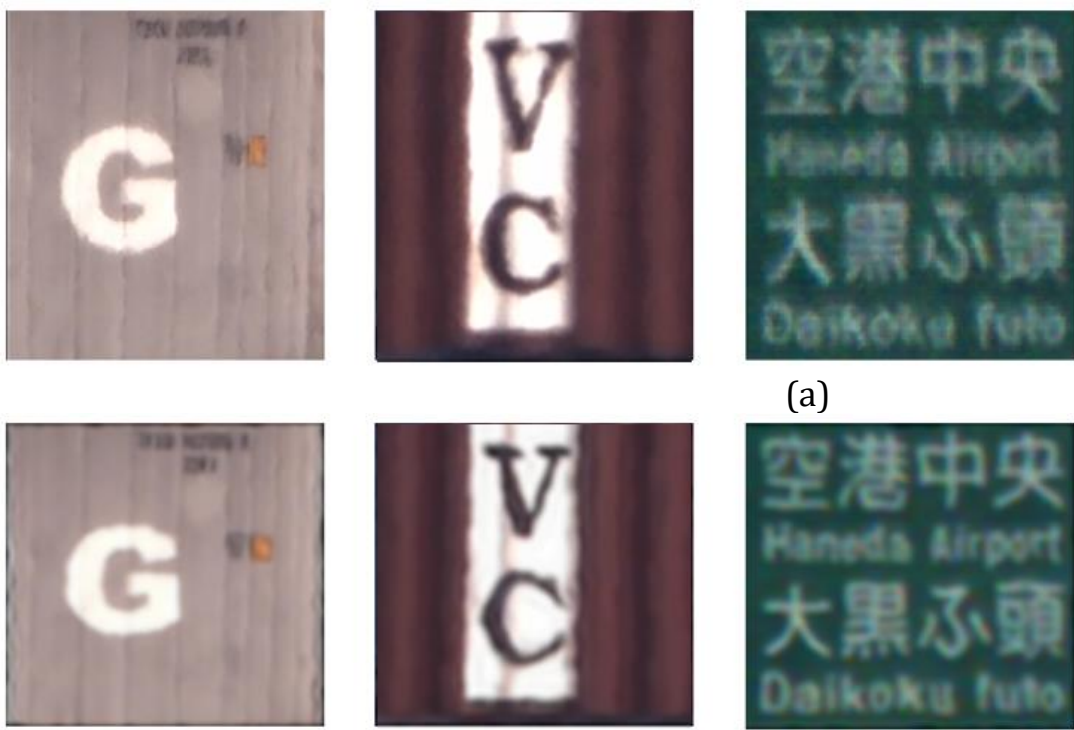

(a)

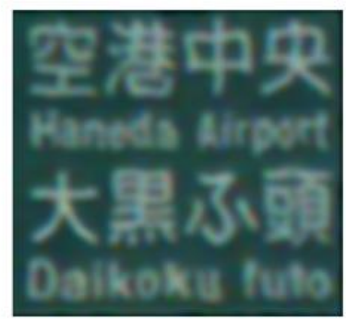

(b)
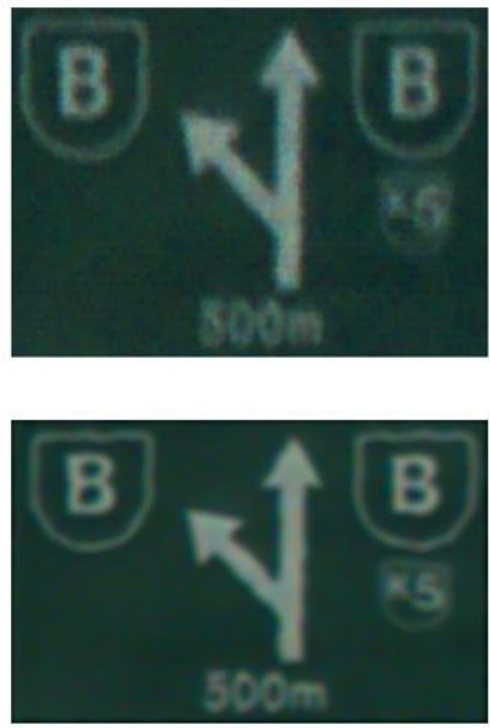

Fig. 7. (a) Real images, (b) Simulation images.

\subsection{Warping by B-spline Function}

In this paper, we use a B-spline function as the warping operator to simulate the deformation by atmospheric turbulence. We first extract landmarks using regular grid. The grid size is set as $15 \times 15$ experimentally for simulating images with the target in $3.2 \mathrm{~km}$. The horizontal and vertical displacements of each landmark are randomly generated according to the normal distributions with $\sigma_{\mathrm{x}}$ and $\sigma_{\mathrm{y}}$, respectively, which are measured from real image data as shown in Fig. 4(b) and Fig. 5. For simulating images with the target in $3.2 \mathrm{~km}$, the measured $\sigma_{\mathrm{x}}$ and $\sigma_{\mathrm{y}}$ are 1.61pixel and 1.27 pixel respectively. Once the displacements of landmarks are determined, the horizontal and vertical displacements of other pixels (non-landmarks) are calculated by the use of B-spline [12] based on the displacements of landmarks.

\subsection{Blurring by Gaussian Blur Kernel}

By analyzing about 300 initial frames, it is easy to draw a conclusion that the degree of blur for each frame is not same due to very far photography distance and extremely complex change of atmospheric turbulence. Fig. 6 shows typical initial example images of 300 continuous frames from a video, and comparatively clear images (Fig. 6 up) and completely unclear images (Fig. 6 bottom) appear randomly. That gives a good explaination about why we can improve image quality using frame selection based super-resolution [7]. In our proposed method, we set the parameters of Gaussian blur kernel (standard deviation) empirically according to real frames. A small part of initial frames are comparatively clear or completely unclear, in consequence the Gaussian blur kernel with size $15 \times 15$ is determined randomly, but obey normal distribution with a mean of 3 and a standard deviation of 1.

\section{Experimental Results}

Fig. 7 shows the degraded original images (Fig. 7(a)) and the simulated images (Fig. 7 (b)) based on reconstructed images using [7]. From visual observation, there was almost no difference between the real deteriorated images and the simulation results, and it was confirmed that there was no unnatural images in the simulations as compared with the real degraded image.

Fig. 8 and Fig. 9 show the results about simulated time-series image data (typical 5 frames) according to 

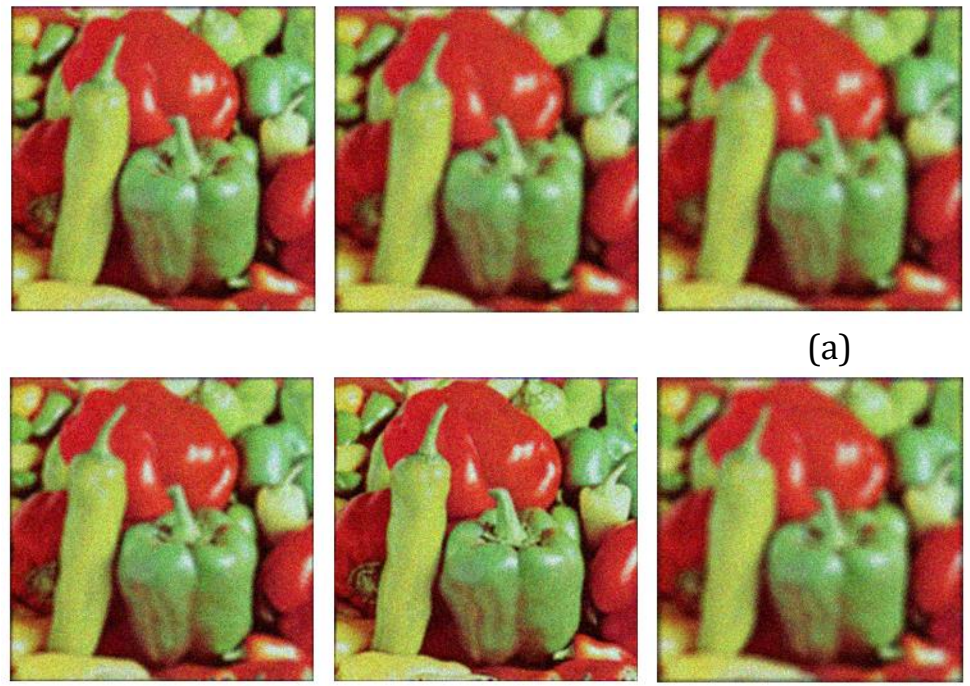

(b)
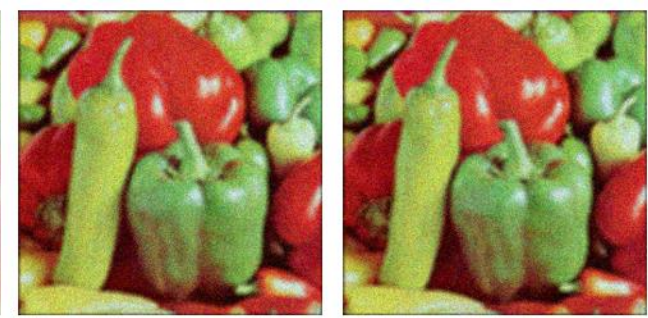

(a)
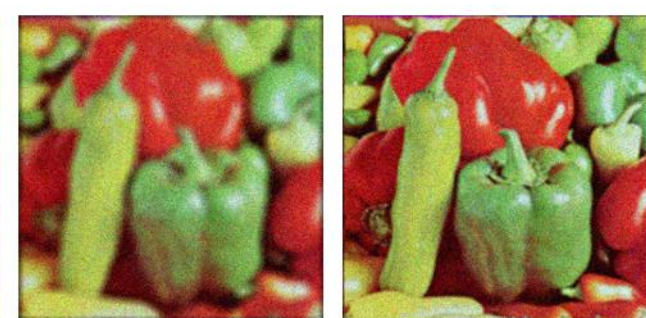

Fig. 8. Simulation results with different photography distances: (a) $3.2 \mathrm{~km}$, and (b) $23 \mathrm{~km}$.
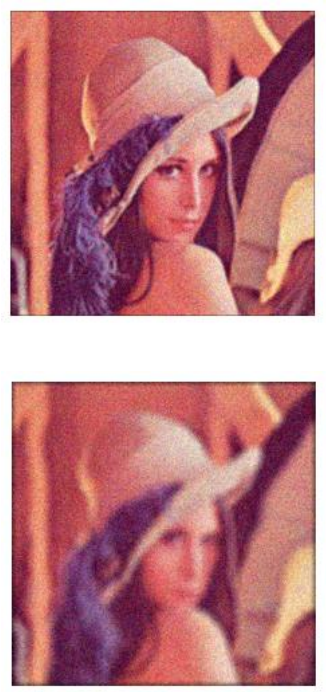
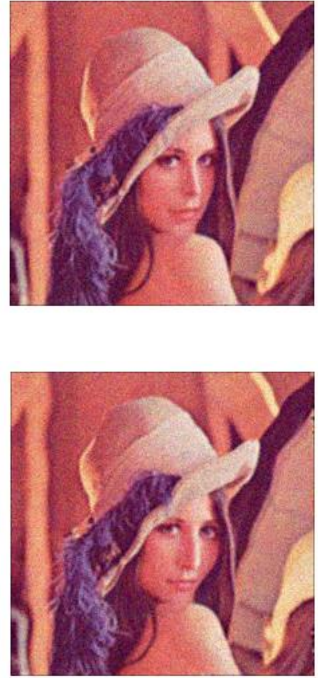

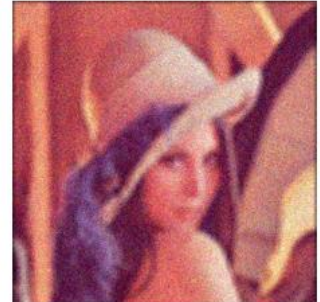

(a)

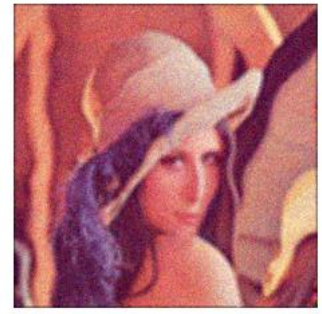

(b)
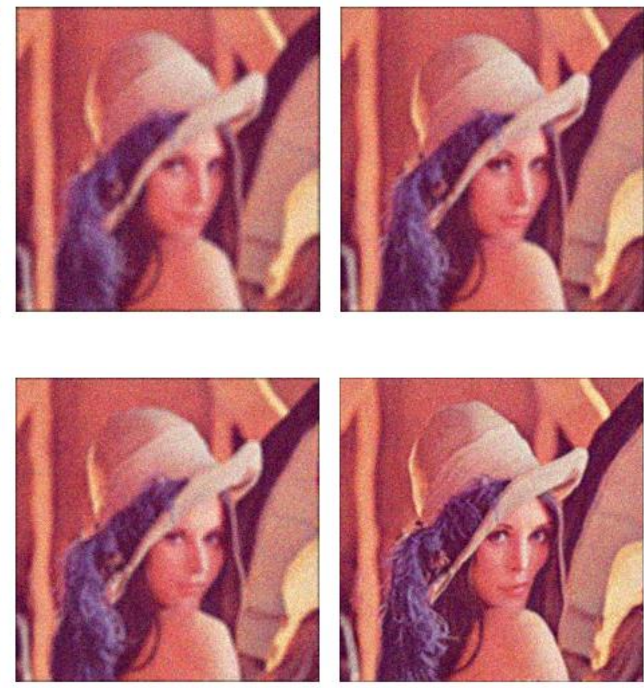

Fig. 9. Simulation results with different photography distances: (a) $3.2 \mathrm{~km}$, and (b) $23 \mathrm{~km}$.

parameters get from real image about the target in 3.2km (Fig. 8(a) and Fig. 9(a)) and 23km (Fig. 8(b) and Fig. 9(b)). The generated $23 \mathrm{~km}$ images have a larger distortion than the $3.5 \mathrm{~km}$ images, which is consistent with the actual observed data.

\section{Conclusion}

In this paper, we estimate the relationship between atmospheric turbulence and photographing distance using deformation parameters computed from real time-series image data. The linear relationship (model) between the atmospheric fluctuation (the standard deviation) and the photographing distance has been found and established. By using our established model, we can simulate the distorted image by atmospheric turbulence. Our simulation model is easier to implement than conventional methods and suitable for quantitative evaluation of image quality improvement method. In our future work, we are going to collect more images with photographing distance from $10 \mathrm{~km}$ to $20 \mathrm{~km}$ for establishing a higher accurate accuracy. 


\section{References}

[1] Bay, H., Ess, A., Tuytelaars, T., \& Van Gool, L. (2008). Speeded-up robust features (SURF). Computer Vision and Image Understanding, 110(3), 346-359.

[2] Canon Global News, (September 2015).

[3] Du Bosq, T. W., \& Repasi, E. (2015). Detector integration time dependent atmospheric turbulence imaging simulation. Proceedings of SPIE, Vol. 9452 (p. 94520B).

[4] Hartley, R., \& Zisserman, A. (2003). Multiple View Geometry in Computer Vision. Cambridge University Press.

[5] Knox, K. T., \& Thompson, B. J. (1974). Recovery of images from atmospherically degraded short-exposure photographs. The Astrophysical Journal, 193, L45-L48.

[6] Li, D., Mersereau, R. M., \& Simske, S. (2007). Atmospheric turbulence-degraded image restoration using principal components analysis. IEEE Geoscience and Remote Sensing Letters, 4(3), 340-344.

[7] Li, Y., Iwamoto, Y., Ogawa, K., \& Chen, Y. W. (2017). Multi-frame super resolution using frame selection and multiple fusion for 250 million pixel images. Proceedings of IEEE International Conference on Consumer Electronics 2018.

[8] Massey Jr, F. J. (1951). The Kolmogorov-Smirnov test for goodness of fit. Journal of the American Statistical Association, 46(253), 68-78.

[9] Mao, Y., \& Gilles, J. (2012). Non rigid geometric distortions correction-application to atmospheric turbulence stabilization. Inverse Problems and Imaging, 6(3), 531-546.

[10] Ng, P. C., \& Henikoff, S. (2003). SIFT: Predicting amino acid changes that affect protein function. Nucleic Acids Research, 31(13), 3812-3814.

[11] Schwartzman, A., Alterman, M., Zamir, R., \& Schechner, Y. Y. (2017). Turbulence-induced 2d correlated image distortion. Proceedings of 2017 IEEE International Conference on Computational Photography (ICCP) (pp. 1-13). IEEE.

[12] Seo, M., Chen, Y. W., \& Aoki, H. (2011). Automatic transformation of KOGAO (Small face) based on fast b-spline approximation. Journal of Information Hiding and Multimedia Signal Processing, 2(2), 192-203.

[13] Shimizu, M., Yoshimura, S., Tanaka, M., \& Okutomi, M. (June 2008). Super-resolution from image sequence under influence of hot-air optical turbulence. Proceedings of IEEE Conference on Computer Vision and Pattern Recognition (pp. 1-8). IEEE.

[14] Torr, P. H., \& Zisserman, A. (2000). MLESAC: A new robust estimator with application to estimating image geometry. Computer Vision and Image Understanding, 78(1), 138-156.

[15] Totsuka, H., Tsuboi, T., Muto, T., Yoshida, D., Matsuno, Y., Ohmura, M., \& Inoue, S. (January 2016). 6.4 An APS-H-Size 250Mpixel CMOS image sensor using column single-slope ADCs with dual-gain amplifiers. Proceedings of 2016 IEEE International Solid-State Circuits Conference (ISSCC) (pp. 116-117). IEEE.

[16] Zhu, X., \& Milanfar, P. (2010). Image reconstruction from videos distorted by atmospheric turbulence. Visual Information Processing and Communication, 7543(1), 75430S.

[17] Zhu, X., \& Milanfar, P. (2013). Removing atmospheric turbulence via space-invariant deconvolution. IEEE Transactions on Pattern Analysis and Machine Intelligence, 35(1), 157-170.

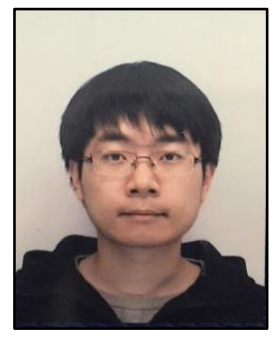

Yinhao Li received a B.E. degree in 2013 from Southeast University Chengxian College, Nanjing, China. Now he is a graduate student in Graduate School of Information Science and Engineering, Ritsumeikan University, Japan. His research interests include image processing and analysis, super-resolution and deep learning. 


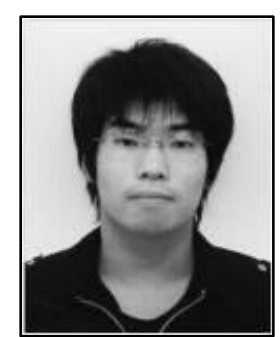

Yutaro Iwamoto received the B.E. and M.E., and D.E. degree from Ritsumeikan University, Kusatsu, Japan in 2011 and 2013, and 2017, respectively. He is currently an Assistant Professor at Ritsumeikan University, Kusatsu, Japan. His current research interests include medical image processing and computer vision, and deep learning.

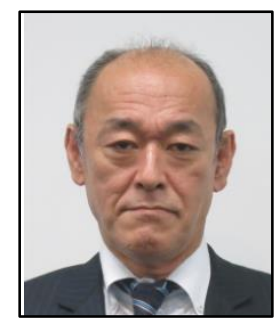

Kazuhisa Ogawa He received a M.E. degree in 1984 from Hokkaido University of Industry, Sapporo, Japan. He is currently a manager of Device Technology Development Headquarts, Canon Inc., Kanagawa, Japan.

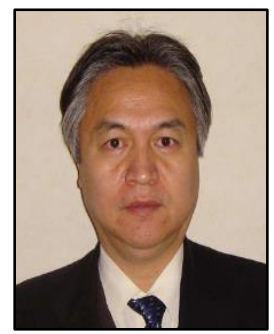

Yen-Wei Chen received a B.E. degree in 1985 from Kobe Univ., Kobe, Japan, a M.E. degree in 1987, and a D.E. degree in 1990, both from Osaka University, Osaka, Japan. From 1991 to 1994 , he was a research fellow with the Institute for Laser Technology, Osaka. From October 1994 to March 2004, he was an associate Professor and a professor with the Department of Electrical and Electronic Engineering, University of the Ryukyus, Okinawa, Japan. He is currently a professor with the college of Information Science and Engineering, Ritsumeikan University, Kyoto, Japan. He is also a chair professor with the college of Computer Science and Technology, Zhejiang University, China. He is an associate Editor of International Journal of Image and Graphics(IJIG) and an associate Editor of the International Journal of Knowledge based and Intelligent Engineering Systems. His research interests include pattern recognition, image processing and machine learning. He has published more than 200 research papers in these fields. 